\title{
Antibiotic prophylaxis in TURP: a prospective analysis concerning antibiotic stewardship and a potential reduction of antibiotic use in TURP
}

\author{
E. Baten ${ }^{1,2,4} \cdot$ F. Van Der $\mathrm{Aa}^{2} \cdot$ C. Orye ${ }^{2} \cdot$ R. Cartuyvels ${ }^{1} \cdot$ I. Arijs $^{3} \cdot$ K. van Renterghem ${ }^{1,2,3}$
}

Received: 11 October 2018 / Accepted: 5 February 2019

○) Springer-Verlag GmbH Germany, part of Springer Nature 2019

\begin{abstract}
Purpose Antibiotic prophylaxis is standard procedure in transurethral resection of the prostate (TURP). We evaluated the necessity of antibiotic (AB) prophylaxis in TURP due to increasing microbial antibiotic resistance.

Methods This is a prospective cohort study of 506 patients. Only patients with a pre-operative catheter/pyuria received ABprophylaxis. Urine analysis (pre-operative, at discharge, and 3 week post-operative) was performed next to an analysis of the blood culture/irrigation fluid and of the resected prostatic tissue. Statistical analysis was performed using Fisher's exact test. Results 67/506 (13.2\%) patients received prophylactic antibiotics. 56/67 (83.5\%) patients had a pre-operative catheter and $11 / 67(16.4 \%)$ had pre-operative pyuria in which a fluoroquinolone-resistance (FQ-R) rate of $69.2 \%$ in Escherichia coli $(E C)$ was observed.

Clinical infectious symptoms were present in 13/439 (2.9\%) patients without antibiotic prophylaxis; 12/439 (2.7\%) patients had uncomplicated fever $\left(<38.5^{\circ}\right)$ during or after hospitalization and only $1 / 439$ patient $(0.2 \%)$ was high degree fever $\left(>38.5^{\circ}\right)$ observed.

Uncomplicated fever developed in 7/67 (10.4\%) patients who did receive AB-prophylaxis.

FQ-R was observed in $60 \%$ of the positive urine cultures at discharge and in $53.8 \% 3$ week post-operatively.

Conclusions Our data show a low infectious complication rate $(2.9 \%)$ in patients without a pre-operative catheter or pyuria,undergoing TURP without AB-prophylaxis. These findings might question the current use of AB prophylaxis in TURP in patients without a pre-operative catheter or pyuria, in times of antibiotic stewardship due to the high rate of microbial-resistance in our population.
\end{abstract}

Keywords TURP $\cdot$ Antibiotics $\cdot$ Microbial resistance $\cdot$ Bacteriuria

\section{Introduction}

Transurethral resection of the prostate (TURP) is one of the most performed urological and even surgical procedures in men [1]. It can be considered as the gold standard in the surgical treatment of $\mathrm{BPH}$, but has some limitations as irritative micturition, prolonged hematuria, need for transfusions, and urinary tract infections.

\footnotetext{
E. Baten

Jessa Hospital, Stadsomvaart, 3050 Hasselt, Belgium

2 UZ Leuven, Herestraat, 3000 Leuven, Belgium

3 U Hasselt, Martelarenlaan 45, 3050 Hasselt, Belgium

4 Leuven, Belgium
}

Post-operative bacteriuria and urinary tract infections have been reported in up to $6 \%$ of the patients and additional high degree fever $\left(38.5^{\circ}\right)$ could develop in $1 \%$ up to $4 \%$ of men undergoing TURP. Risk factors associated with postoperative bacteriuria and urinary tract infection are considered prolonged operating time, a pre-operative catheter, and prolonged post-operative catheter [1].

TURP can be considered as a clean-contaminated procedure, and the use of antibiotic $(\mathrm{AB})$ prophylaxis to reduce bacteriuria/bacteremia and clinical infectious symptoms is recommended by the EAU/AUA guidelines (level 1A evidence). These recommendations, however, are mainly based on three studies (a meta-analysis, systematic review, and randomized-controlled trial), all published between 2002 and 2005 [1-3]. 
The current guidelines recommend a single dose of trimethoprim \pm sulphamethoxazole or aminopenicillin/betalactamase inhibitor or cephalosporin group 2 or 3 [4].

Adherence to the EAU guidelines is not universal, and the extended duration of antibiotic administration after a surgical procedure without any infective indication encourages the development of multidrug-resistant organisms, poorer clinical outcomes, and higher treatment costs [5].

Recent reports have shown that adherence to these guidelines reduced antibiotic usage without increasing post-operative infection rate and lowered the prevalence of resistant uropathogens [5].

Our study was initiated to investigate the safety of omitting antibiotic prophylaxis in a subset of patients undergoing TURP to reduce the impact on microbial resistance, since even a single dose of antibiotics can induce microbial resistance as shown by Wagenlehner et al. [6] and because a total reduction in antibiotic consumption remains the 'holy grail' in antibiotic stewardship [7].

A prospective, observational study was set up to evaluate the safety of AB-prophylaxis (AB-P) in patients undergoing TURP without pre-operative pyuria/catheter and to evaluate the antibiotic resistance rates of uropathogens.

\section{Materials and methods}

Approval was obtained from the Ethical Committee of Jessa Hospital Hasselt (B243201733479) despite the current guidelines because of the increasing importance of antibiotic stewardship in which a responsible reduction of antibiotics should be obtained and as such further investigated.

A prospective study was initiated from 08/2008 until 09/2015. All patients underwent TURP for bothersome, pharmaco-therapy refractory obstructive LUTS (lower urinary tract symptoms).

Demographics as PSA (prostate specific antigen), IPSS (International Prostate Symptom Score), prostate volume (measured by ultrasound), and uroflow investigation were analyzed together with pre-operative investigations as cystoscopy, prostate biopsies, or urodynamic investigation. The pre-operative catheterization status and if the TURP was primary or re-do surgery were also noted. Mid-stream urine samples were collected pre-operatively at arrival in the hospital and investigated for pyuria.

Only patients with a pre-operative indwelling transurethral (TU) or suprapubic (SP) catheter or pre-operative pyuria [ $>100$ white blood cells (WBC)/ml of urine)] received prophylactic antibiotics, because these are known risk factors for developing post-operative clinical infectious symptoms.

Single-dose fluoroquinolones were empirically administered or alternative antibiotics in case of antibiotic resistance when the previous urinary cultures were available. Our choice for fluoroquinolones was based on the Belgian national antibiotic guidelines (BAPCOC) which recommend a single dose of fluoroquinolones as AB-P in TURP [8].

All TURP procedures were performed under spinal anesthesia by the same surgeon using monopolar cautery and all patients had a post-operative rinsing catheter, which was generally removed after 2 days. Surgical variables as operating time, post-operative sodium- and hemoglobin levels, and amount of resected prostate tissue were analyzed. No antibiotics were systematically prescribed after the removal of the transurethral catheter and most patients went on discharge at day 2. All clinical infectious symptoms were analyzed with a distinction between uncomplicated fever $\left(<38.5^{\circ}\right)$ and high degree fever $\left(>38 \cdot 5^{\circ}\right)$. All patients were seen after 3 weeks at the follow-up consultation.

Mid-stream urine samples were collected at discharge and after 3 weeks at the follow-up consultation. A post-operative blood- and irrigation fluid sample and a piece of resected prostate tissue were collected for microbial cultivation. All collected samples were investigated by the same experienced microbiologist, and the level of microbial significance was scored from 0 to 2 ; in which 0 meant no significant growth and 2 meant a significant microbial growth $\left(>10^{5} \mathrm{CFU}\right)$. Positive cultures in the absence of clinical symptoms were not treated with antibiotics.

Statistical analysis was performed with the two-sided Fisher's exact test to investigate the significance of the deviation of the null hypothesis for categorical variables. The R Project for statistical computing was used for our calculations.

\section{Results}

\section{Surgical data}

506 consecutive patients, undergoing TURP, were included in our study. Mean PSA was $4.54 \mathrm{ng} / \mathrm{ml} \pm 4.3$, mean IPSS $16.1 \pm 7.2$, mean prostate volume (measured by rectal ultrasound) $38.6 \mathrm{~g} \pm 22.3$, mean peak flow $10.4 \mathrm{ml} / \mathrm{min} \pm 7.9$, and mean residual volume $103.7 \mathrm{ml} \pm 154.481 / 506(95 \%)$ patients had a pre-operative cystoscopy, 316/506 (64\%) had urodynamics, and 217/506 (43\%) had prostate biopsies (Table 1).

The high rate of prostate biopsies is due to our patient population, consisting of patients with a high PSA and negative clinical examination/rectal ultrasound for prostate cancer before the current wide-spread use of MRI. Patients received a single dose of FQ as prophylaxis and the TURP was performed at least 3 months after the biopsies.

The mean operating time was $31.2 \mathrm{~min} \pm 11.5$ and the median hospital stay was 2 days. Mean post-operative 
Table 1 Patients' demographics and pre-operative investigations

Pre-operative demographics

Mean PSA

$4.5 \mathrm{ng} / \mathrm{ml} \pm 4.3$

Mean IPSS

$16.1 \pm 7.2$

Mean prostate volume

$38.6 \mathrm{~g} \pm 22.3$

Mean peak flow

$10.4 \mathrm{ml} / \mathrm{min} \pm 7.9$

Mean residual volume

$103.7 \mathrm{ml} \pm 154$

Cystoscopy

481/506 (95\%)

SP/TU catheter

$58 / 506(10.7 \%)$

Prostate biopsies

$217 / 506(43 \%)$

Urodynamics

$316 / 506(64 \%)$

$S P$ suprapubic, $T U$ transurethral, IPSS International Prostate Symptom Score

natrium was $138.8 \pm 3.1$, mean post-operative hemoglobin $14.7 \mathrm{mg} / \mathrm{dl} \pm 7.8$, and the mean amount of resected tissue weight was $34.9 \mathrm{~g} \pm 24.9$.

\section{Antibiotic prophylaxis}

Only patients with a pre-operative catheter or pyuria received AB prior TURP. 67/506 (13.2\%) patients received prophylactic AB of which 11/67 (16.4\%) had pre-operative pyuria and $56 / 67(83.5 \%)$ had a pre-operative catheter.

$60 / 67(89.5 \%)$ patients received prophylactic ciprofloxacin, 4/67 (5.9\%) patients amoxicillin with clavulanic acid, and $3 / 67(4.5 \%)$ patients meropenem due to an extendedspectrum beta-lactamase E. coli (ESBL).

We observed clinical infectious symptoms in 13/439 (2.9\%) patients who had no antibiotic prophylaxis and in 7/67 (10.4\%) patients who received prophylaxis during hospitalization, resulting in a statistically significant difference ( $p$ 0.001) (Fig. 1). No significant correlation was observed between post-operative infection and a pre-operative catheter $(p 0.16)$.

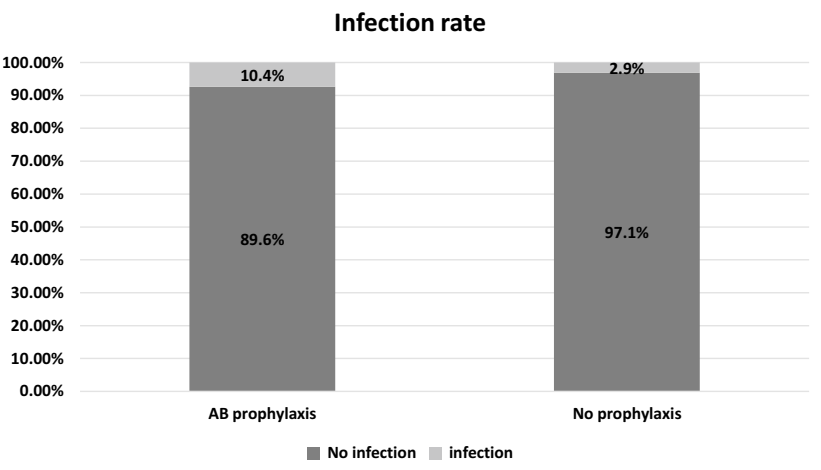

Fig. 1. Infection rates in patients with or without $\mathrm{AB}$-prophylaxis show a significant difference between both groups ( $p 0.001)$
$37 / 506(7.3 \%)$ patients were discharged with AB, 28/37 (76.7\%) patients received ciprofloxacin, 7/34 (20.6\%) amoxicillin with clavulanic acid, and 2/37 (5\%) sulfamethoxazole + trimethoprim. 10/506 (1.8\%) patients had fever at home, requiring re-hospitalization in 3/506 $(0.6 \%)$ patients.

Most common bacteria in the pre-operative urine culture were E. coli (EC) (28.2\%), K. pneumoniaeloxytoca $(21.7 \%)$ and E. faecalis (EF) (19.5\%). Fluoroquinolone resistance (FQ-R) amounted $69.2 \%$ in EC and $40 \%$ in Klebsiella (Fig. 2). A significant association between pre-operative bacteriuria and a pre-operative catheter was observed $(p<0.001)$.

Blind blood cultures were taken in every patient at the recovery unit and $31 / 506(6.1 \%)$ patients had a significant bacteremia with EC (19.4\%) and hemolytic streptococci (19.4\%) as main pathogens. FQ-R was 50\% in EC and $0 \%$ in $\mathrm{EF}$.

A sample of the irrigation fluid was taken upon arrival at the ward and $24 / 506(4.7 \%)$ patients had a positive culture of the irrigation fluid. Causal pathogens were EC (29.2\%), and EF (25\%). FQ-R was $85.7 \%$ in EC and $0 \%$ in $\mathrm{EF}$.

$58 / 506(11.4 \%)$ patients had a significant bacteriuria at time of discharge. Main pathogens were EF (29.8\%), Klebsiella (13.5\%), and EC (13\%\%). FQ-R was $75 \%$ in EC and $60 \%$ in Klebsiella.

Prostate tissue culture showed a significant infection in $37 / 506(7.3 \%)$ patients with EF (37.8\%) and Staphylococci (16.2\%) as main pathogens. FQ-R was $100 \%$ in EC and $7.6 \%$ in $\mathrm{EF}$.

$36 / 506(7.1 \%)$ patients had a significant bacteriuria 3 week post-operatively with EC as causal pathogen in $36.1 \%$ and $\mathrm{EF}$ in $44.4 \%$. FQ-R was $53.8 \%$ in EC, and $25 \%$ in $\mathrm{EF}$, with another $25 \%$ intermediate-resistance rate.

Only one patient (3\%) with a clinical infection had diabetes mellitus type 2 .

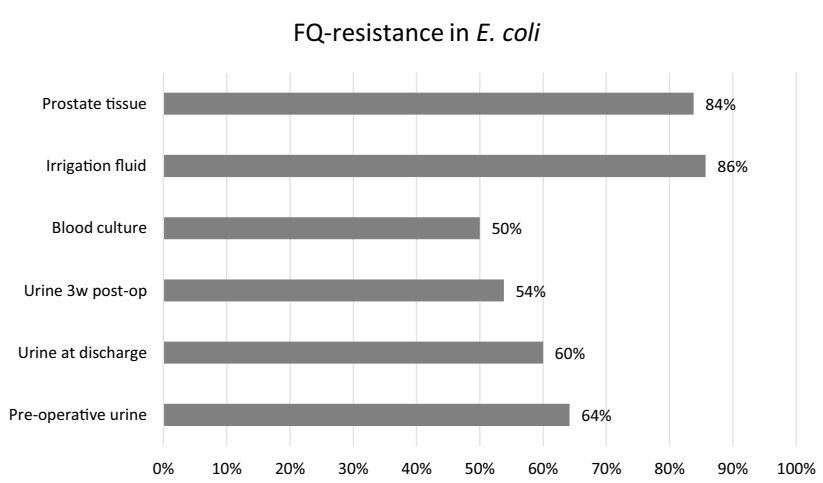

Fig. 2 FQ resistance in E. coli 


\section{Discussion}

The EAU and AUA guidelines [2,3] strongly recommend the use AB-prophylaxis prior TURP (level 1A evidence) to decrease microbial and clinical infectious symptoms. These recommendations, however, are mainly based on three studies (a meta-analysis, systematic review, and randomized-controlled trial), all published between 2002 and 2005.

Berry et al. described a significant decrease in bacteriuria and clinical septicemia incidence in their meta-analysis published in 2002 [2]. They state that if 1.000 patients would be given AB-prophylaxis prior TURP, bacteriuria could be avoided in 175/1000 (17.5\%) patients, while clinical septicemia could be prevented in $9 / 1000(0.9 \%)$ to 20/1000 (2\%) patients.

Qiang et al. published a systematic review in 2005, including 28 studies which were published between 1966 to 2002 , stating that antibiotic prophylaxis was significantly more effective than placebo in reducing postoperative TURP complications [1]. They described a risk difference for post-TURP bacteriuria in men receiving AB-prophylaxis vs placebo from 26.2 to $9.2 \%$. Only 6 of the 28 studies in this systematic review showed a decrease in post-operative high degree fever $\left(>38.5^{\circ}\right)$ with a risk difference of -0.11 . They state that these benefits of antibiotic prophylaxis should be weighed against a 2.6 -fold increase in adverse events in the antibiotic group, costs, and the potential for the development of antibiotic resistant organisms.

A large randomized-controlled trial was published by Wagenlehner et al. in 2005 [3], in which they compared single-dose AB-prophylaxis [levofloxacin or trimetho$\mathrm{prim} / \mathrm{sulfamethoxazole}$ (TMP/SMZ)] versus a control group (2:2:1 ratio). Their goals were bacteriuria after 5-7 days, 3-5 weeks and the overall complication rate. 376 patients were included of which $74(19.7 \%)$ received no antibiotics. No statistic significant difference was observed between the total bacteriuria rate between the $\mathrm{AB}$ and control groups after 5-7 days and 3-5 weeks. It is unclear if post-operative bacteriuria correlates with the complication rate and Wagenlehner et al. do not recommend to treat post-operative bacteriuria as such [5]. An increase in the post-operative $\mathrm{AB}$ use was noted between the $\mathrm{AB}$ and control groups, but no clear criteria for this $\mathrm{AB}$ use were objectivated. The total complication rate at weeks 3-5 was also not statistical significant between the $\mathrm{AB}$ and control groups. An important antibiotic resistance of $37 \%$ to levofloxacin and $43 \%$ to TMP/SMZ was noted.

These studies showed a small benefit for the use of antibiotic prophylaxis to reduce post-operative bacteriuria and urinary tract infections, but also state that the use of prophylaxis should be weighed against the increase of adverse events, costs, and potential for developing antibiotic resistant organisms. Currently, antibiotic stewardship is recommended due to the increasing antibiotic resistance and the increasing costs in medical care.

Only patients with a pre-operative catheter or pyuria received AB-prophylaxis prior to TURP, and we observed clinical infectious symptoms in 13/439 (2.9\%) patients who had no antibiotic prophylaxis and in 7/67 (10.4\%) patients who received prophylaxis which resulted in a significant difference ( $p$ 0.001). The high rate of infections in the AB group can be explained because of the higher risk factors for infection (pre-operative pyuria/catheter) and the surprisingly high antibiotic resistance rates. Microbial directed AB-P was only given if the previous cultures showed fluoroquinoloneresistance. Pre-operative cultures were collected shortly before TURP due to practical reasons, and as such, results of the urinary cultures were unavailable at time of surgery, so an inadequate prophylaxis was mostly given in a high-risk population for infections. One of the main reasons for the high FQ-R rate could be the high rate of prostate biopsies in our population and subsequent microbial resistance development for fluoroquinolones which were used as prostate biopsy prophylaxis, as Wagenlehner et al. already showed in 2000 that even a single dose of ciprofloxacin increases antimicrobial resistance rates in $E$ coli from 3 to $12 \%$ ( $p=$ 0.052) [4].

In the group of patients without AB-prophylaxis and clinical, infectious symptoms (13/439 or $2.9 \%), 12 / 439(2.7 \%)$ patients had uncomplicated fever during or after hospitalization and only $1 / 439$ patient $(0.2 \%)$ was high degree fever or septicaemia observed.

Our clinical infectious complication rate is low and comparable with findings in the literature [9]. Our data suggest that omitting AB-prophylaxis prior TURP in patients without pre-operative indwelling catheter or pyuria does not result in an increase of clinical infections and can be considered safe in this subset of patients undergoing TURP.

We experience in our daily practice an increasing rate of antibiotic resistance and to fluoroquinolones in particular. These findings are reflected in our high rate of AB-resistance in our microbial cultures with a remarkable FQ-resistance in E. coli (Fig. 2), which can result in multi-resistant bacteria which cause hard-to-treat infections with high morbidity/ mortality and additional health care expenses.

Most AB are simply ineffective as prophylaxis in TURP due to the high rate of AB-resistance as shown in our study where many patients received empiric fluoroquinolones despite fluoroquinolone-resistant uropathogens, because the results of the urinary cultures were not available at time of surgery.

We recommend as such collecting a pre-operative urinary sample 3 or 4 days before TURP instead upon arrival 
in the hospital like in our study. The timing of the preoperative urine sampling is important, since many patients with FQ-R uropathogens received FQ in our study, because the results of the cultures (which take 2-3 days) were not available at the time of surgery.

In case of a positive urinary culture (asymptomatic bacteriuria), a directed AB-prophylaxis should be used. As such, only a selected population of patients undergoing TURP receives microbial culture-directed antibiotics as prophylaxis instead of all the patients receiving empiric antibiotics.

Together with the increasing problem of resistance, bothersome concerns arise due to the potential side-effects of FQ.

The FDA (Food and Drugs Administration) recently approved changes to the labels of fluoroquinolone antibacterial drugs for systemic use and revised the Boxed Warning (FDA's strongest warning), because these medicines are associated with disabling and potentially permanent side effects of the tendons, muscles, joints, nerves, and central nervous system [10].

Recently, the Belgian government has made severe regulations to discourage the general use of fluoroquinolones and to restrict its use only for microbial targeted, urological infections [11], but the national guidelines still recommend empiric fluoroquinolones as prophylaxis in TURP.

These new regulations were set up to reduce the excessive use of antibiotics to decrease its financial impact on medical care and to reduce the antibiotic resistance in Belgium.

Since Berry's meta-analysis 16 years earlier, we are increasingly encouraged by the government to reduce our expenses in health care. TURP is one of the most performed urological procedures, and as such, a major cost-saving impact could be expected if we are able to reduce the procedure-related costs.

Additional pre-operative urine sediment, however, could be regarded as an added cost in some centers, but, as only the positive sediments will be sent for microbial urine culture analysis, this will not result in a major cost. The benefit of a microbial culture in case of a post-operative infection is invaluable due to the high antibiotic resistance rates, which limit the use of empiric antibiotics.

We strongly suggest to reduce the use of FQ as TURP prophylaxis and recommend, when AB-prophylaxis is opportune, to use pre-operative urine culture-directed $\mathrm{AB}$ instead.

Our data are promising and suggest the safety of omitting AB-prophylaxis in patients without a pre-operative catheter or pyuria. Despite our large number of consecutive patients (506 patients), good methodology (prospective study, same surgeon, and same team of microbiologists) and extensive microbial analysis, our single-center, cohort study is limited, because it lacks randomization and is single-centered.
We believe that further research is needed with a multicenter, randomized-controlled trial to investigate and confirm our findings.

\section{Conclusion}

A responsible use of antibiotics is the key point of antibiotic stewardship and a reduction of $\mathrm{AB}$ use should be obtained whenever safe and feasible. Only patients with pre-operative pyuria or catheter received AB-prophylaxis prior TURP in our large, prospective study. A low number of clinical infections in our patients undergoing TURP without AB-prophylaxis and a remarkable fluoroquinolone-resistance rate in $E$. coli were observed, which resulted in a higher amount of infections in the $\mathrm{AB}$ group who received mostly inadequate, empiric fluoroquinolones according to national guidelines. We believe that it is safe to omit AB-prophylaxis in patients undergoing TURP without pre-operative pyuria or a catheter and that the disadvantages of empiric $\mathrm{AB}$-prophylaxis $(\mathrm{AB}$ resistance, side effects, and financial costs) outweigh the potential benefit of reducing clinical infections.

We recommend, however, to collect a pre-operative urinary sample 3 or 4 days before TURP, so a microbial culture-directed AB-prophylaxis can be used in case of a positive culture instead of empiric antibiotic prophylaxis in every patient undergoing TURP. Additional randomized-controlled trials are needed to confirm our findings.

Author contributions EB: protocol/project development; data collection or management; data analysis; manuscript writing/editing. FA: manuscript writing/editing. CO: data collection or management. RC: protocol/project development. IA: manuscript writing/editing. KR: protocol/project development; data collection or management; manuscript writing/editing.

\section{Compliance with ethical standards}

Conflict of interest We have nothing to disclose.

Informed consent Informed consent was obtained for every patient.

\section{References}

1. Qiang W, Jianchen W, MacDonald R et al (2005) Antibiotic prophylaxis for transurethral prostatic resection in men with preoperative urine containing less than 100,000 bacteria per ml: a systematic review. J Urol 173(4):1175-1181

2. Berry A, Barratt AJ (2002) J Prophylactic antibiotic use in transurethral prostatic resection: a meta-analysis. J Urol 167:571-577

3. Wagenlehner FM, Wagenlehner C, Schinzel S et al (2005) Prospective, randomized, multicentric, open, comparative study on the efficacy of a prophylactic single dose of $500 \mathrm{mg}$ levofloxacin versus $1920 \mathrm{mg}$ trimethoprim/sulfamethoxazole versus a control 
group in patients undergoing TUR of the prostate. Eur Urol 47(4):549-556

4. Bonkat G, Pickard R, Bartoletti R, et al (2017) EAU guidelines on urological infections. Perioperative antibacterial prophylaxis in urology. In: Complete European Association of Urology guidelines. Edn. Presented at the EAU annual congress London 2017, pp 35-9

5. Cai T, Verze P, Bartoletti R et al (2016) Adherence to European Association of Urology guidelines on prophylactic antibiotics: an important step in antimicrobial stewardship. Eur Urol 69(2):276-283

6. Wagenlehner F, Stöwer-Hoffmann J, Schneider-Brachert W, Naber KG, Lehn N (2000) Influence of a prophylactic single dose of ciprofloxacin on the level of resistance of Escherichia coli to fluoroquinolones in urology. Int J Antimicrob Agents 15(3):207-211

7. Gould I (2016) Antibiotic stewardship: what's not to like? Eur Urol 69:284-285

8. Belgian Antibiotic Policy Coordination Committee: richtlijnen voor anti-infectieuze behandelingen in ziekenhuizen. BVKIM 2017.
9. Wolf JS Jr, Bennett CJ, Dmochowski RR et al (2008) Best practice policy statement on urologic surgery antimicrobial prophylaxis. J Urol 179(4):1379-1390. https://doi.org/10.1016/j. juro.2008.01.068

10. Fluoroquinolone antibacterial drugs for systemic use: drug safety communication-warnings updated due to disabling side effects. 07/26/2016.

11. FGOV.be; RIZIV: wijziging terugbetaling antibiotica vanaf 1 mei 2018.

Publisher's Note Springer Nature remains neutral with regard to jurisdictional claims in published maps and institutional affiliations. 